\title{
A modest proposal
}

\author{
Peter E Lipsky
}

In the US, six biologic agents have been approved for the treatment of patients with rheumatoid arthritis (RA). This remarkable achievement gives patients hope that the adverse effects of this chronic inflammatory disease can be effectively controlled. It is not clear, however, what is the way forward to enable rheumatologists to learn to use these agents optimally.

Traditionally, drugs for RA have become available through the usual regulatory approval mechanism, and then physicians have learned to use them appropriately through investigatorinitiated trials. Rheumatologists have a long history of learning to apply drugs effectively, as was the case with methotrexate. With the advent of biologics, however, this dynamic has changed, and perhaps not in a positive way. Their high cost has sharply limited the possibility of designing and carrying out the investigator-initiated trials that are essential for the rheumatology community to discover the best way of using these agents in individual patients. Specifically, we do not have data on the comparative efficies of the biologics, the appropriate sequencing of agents, the best cotherapies, or the possible combined or staggered use of biologics marketed by different companies.

The high cost of biologics can even dictate how they are used in practice, because it is frequently the third-party insurer, health-plan co-ordinator or governmental agency that decides which patients will receive biologics and when in the course of disease these drugs will be made available. These decisions are commonly based upon criteria established for the entry of patients into clinical trials. As recent evidence suggests that only a minority of RA patients in clinical practice meet entry criteria for clinical trials (Sokka and Pincus [2003] Arthritis Rheum 48: 313), it is remarkable that
Rheumatologists

have a long

history of

learning to

apply drugs

effectively ....

With the advent of biologics, however, this dynamic has changed...

PE Lipsky is the Editor-in-

Chief of Nature Clinical Practice Rheumatology.

\section{Competing interests}

The author declared he has no competing interests.

www.nature.com/clinicalpractice doi:10.1038/ncprheum0223 these standards should be applied to the general population of patients with RA. Because of the high cost of biologics, we are in an unusual situation, in which the pharmaceutical companies, regulatory agencies, insurers and health-plan co-ordinators have acquired remarkable de facto authority over decisions involving the use of these medications in clinical practice. Additionally, there seems to be little opportunity for investigator-initiated studies to provide the necessary evidence to counter-balance these influences.

So, what can be done? Among a number of possible solutions, one stands out as relatively simple and feasible. An international consortium of interested parties could be established to oversee the awarding of grants to support meritorious trials that address clinical questions related to biologic agents. There are numerous professional and patient groups that could contribute expertise to the establishment of this consortium. Importantly, the consortium should be free of conflicts of interest and the marketing influences of the pharmaceutical industry. Funding could be from a small surcharge on the companies marketing biologics, for instance $2 \%$ of gross sales (and investigators should also be allowed access to the therapeutic agents and relevant placebos). This unrestricted support for the consortium should not be a burden to the companies, could be redirected from some of their marketing efforts and in the long run might be valuable to them by expanding their markets appropriately. This sort of effort would provide an appropriate means to address important clinical questions about biologics and to provide the evidence permitting the appropriate application of these potent new agents. 